\title{
Bemerkenswerte, aktuelle Publikationen aus Österreich
}

Osterwalder J, Mathis G, Hoffmann B. New Perspectives for Modern Trauma Management - Lessons Learned from 25 Years FAST and 15 Years E-FAST. Ultraschall in Med 2019 Oct; 40(5): 560-583. doi:10.1055/a-09245455. Epub 2019 Oct 9.

Hirtler L, Rath C, Platzgummer H, Aletaha $D$, Kainberger F. Pseudoerosions of Hands and Feet in Rheumatoid Arthritis: Anatomic Concepts and Redefinition. J Clin Med 2019 Dec 9; 8(12). pii: E2174. doi:10.3390/ jcm8122174.
Sconfienza LM, Adriaensen M, Albano D, Allen G, Aparisi Gómez MP, Bazzocchi A, Beggs I, Bignotti B, Chianca V, Corazza A, Dalili D, De Dea M, Del Cura JL, Di Pietto F, Drakonaki E, Facal de Castro F, Filippiadis D, Gielen J, Gitto S, Gupta H, Klauser AS, Lalam R, Martin S, Martinoli C, Mauri G, McCarthy C, McNally E, Melaki K, Messina C, Mirón Mombiela R, Neubauer B, Olchowy C, Orlandi D, Plagou A, Prada Gonzalez R, Rutkauskas S, Snoj Z, Tagliafico AS, Talaska A,
Vasilevska-Nikodinovska V, Vucetic J, Wilson D, Zaottini F, Zappia M, Obradov M. Clinical indications for image-guided interventional procedures in the musculoskeletal system: a Delphi-based consensus paper from the European Society of Musculoskeletal Radiology (ESSR)-part I, shoulder. Eur Radiol 2019 Sep 16. doi:10.1007/s00330019-06419-x. [Epub ahead of print] 